\title{
Internet des Objets pour l'apprentissage humain
}

\section{Internet of Things for human learning}

\author{
Aymeric Bouchereau', Ioan Roxin ${ }^{1}$ \\ ${ }^{1}$ Laboratoire ELLIADD, Université de Franche-Comté, France, \\ aymeric.bouchereau@edu.univ-fcomte.fr, ioan.roxin@univ-fcomte.fr
}

RÉSUMÉ. L'Internet des Objets se traduit par une multitude d'objets connectés dotés de fonctions avancées pour l'automatisation et l'assistance aux personnes, soulevant des défis techniques, politiques, sociaux et économiques. L'apprentissage est lié à ces défis en plusieurs sens : si l'apprentissage machine contribue au fonctionnement des objets connectés, ces derniers stimulent en retour l'apprentissage humain en diversifiant les pratiques et les outils. Les méthodes d'apprentissage peuvent s'appuyer sur les récents travaux en neurosciences identifiant les ressorts cognitifs de l'apprentissage : l'attention, l'engagement, le retour d'informations et la consolidation. En outre, l'apprentissage s'est vu modifié par l'évolution technologique: après l'imprimerie, l'informatique et les télécommunications, l'Internet des Objets est un nouveau support d'information pour la transmission des savoirs. À partir de trois dimensions d'analyse (données, interfaces et pervasivité), nous proposons une classification des articulations possibles de l'Internet des Objets pour soutenir l'apprentissage. L'étude de cette relation montre que l'Internet des Objets favorise l'exploration et l'expérimentation, la mise en place de situations authentiques et la contextualisation de l'apprentissage.

ABSTRACT. The Internet of Things encompasses a multitude of connected devices with advanced functions for the automation of tasks and assistance to individuals, raising technical, political, social and economic challenges. Learning is linked to these challenges in several ways: the machine learning development contributes to the functioning of connected devices, which in turn stimulate human learning by diversifying practices and tools. Learning methods can lean on recent works in neuroscience that identify cognitive resources for learning, such as attention, engagement, feedback and consolidation. In addition, learning has been modified by technological change: after printing, computing and telecommunications, the Internet of Things is a new information medium for knowledge transmission. Based on three dimensions (data, interfaces and pervasiveness), we propose a classification of possible articulations of the Internet of Things to support learning. The study of this relationship shows that the Internet of Things promotes exploration and experimentation, the setting up of authentic situations and the contextualization of learning.

MOTS-CLÉS. Apprentissage, Apprentissage basé sur l'IdO, Internet des Objets, Objets connectés, Sciences cognitives. KEYWORDS. Cognitives sciences, Connected devices, loT-Based learning, Internet of Things, Learning.

\section{Introduction}

Traduisant le développement de l'Internet des Objets (IdO), la multiplicité des objets connectés accentue l'imbrication du numérique avec le monde physique. Les objets du quotidien deviennent des objets «connectés » avec des fonctions avancées pour l'automatisation de tâches et l'assistance aux personnes. Passant par l'acquisition de capacités similaires aux individus, ces fonctions sont la source de défis techniques complexes-pour acquérir de telles capacités-, mais aussi politiques, sociaux et économiques de par leurs répercussions [SAL 17].

L'apprentissage est une réponse à ces défis, aussi bien en tant qu'activité humaine qu'en tant que processus informatique. Les fonctionnalités des objets connectés tirent leur pertinence et cohérence de systèmes apprenants qui, à partir de grands volumes de données, parviennent à prédire ou à évaluer une situation et agir en conséquence. En même temps, les changements induits rendent essentielle la formation tout au long de la vie pour suivre l'évolution des utilisations et s'adapter aux nouveaux besoins en compétences. Or, la relation entre apprentissage et IdO est bidirectionnelle: si l'apprentissage machine contribue au développement de l'IdO en améliorant les fonctions des objets connectés, l'IdO soutient en retour l'apprentissage humain à travers de nouveaux modes et méthodes d'acquisition des connaissances. En effet, avec l'imprimerie, l'informatique et les télécommunications, les objets connectés sont de nouveaux supports d'informations dont il est nécessaire de saisir les implications et opportunités pour l'apprentissage humain. 
Dans cet article, nous présentons les articulations entre l'IdO et l'apprentissage humain. En premier lieu, nous explicitons les mécanismes de l'apprentissage en traitant les aspects cognitifs et l'évolution technologique jusqu'au développement de l'IdO. En second lieu, nous nous appuyons sur les dimensions caractéristiques de l'IdO-données, interfaces et pervasivité-afin d'étayer une classification des applications et activités possibles pour apprendre. Enfin, nous terminons par une mise en perspective de la relation entre IdO et apprentissage, aux niveaux humain et informatique.

\section{1. «Apprendre est l'essence de la vie "}

Apprendre est une activité menée par une personne en vue d'acquérir ou approfondir un savoir ou un savoir-faire. Pour Jiddu Krishnamurti il existe deux apprentissages: l'accumulation de connaissances et l'apprentissage présent et continu [KRI 10]. Il suggère qu'apprendre ne se limite pas à l'acquisition de connaissances, mais est un état d'esprit permanent où l'on observe et explore le monde. Edgar Morin ajoute que l'apprentissage ne s'apparente pas seulement à un processus de reconnaissance ou de découverte, mais à la combinaison des deux : le connu et l'inconnu [MOR 92] (p. 61). Morin rappelle également que nous vivons une époque stimulante sur le plan de la connaissance, celle-ci étant constituée de multiples découvertes et résolutions de problèmes [MOR 92] (p. 1180). Il pense que ce qui est vital pour le principe organisateur de la connaissance «ce n'est pas seulement d'apprendre ", de réapprendre, de désapprendre, "mais de réorganiser notre système mental pour réapprendre à apprendre »[MOR 08] (p. 44). Aujourd'hui, l'évolution des technologies de l'information et de la communication (TIC) a démultiplié les possibilités de captation, de partage et de production de l'information, avec des connaissances plus fines sur les mécanismes de l'apprentissage et ses ressorts cognitifs.

\subsection{Mécanismes de l'apprentissage}

L'étude des mécanismes de l'apprentissage a donné lieu à plusieurs courants théoriques. Le behaviorisme, par exemple, se base sur le modèle stimuli-réponse et considère essentiellement le comportement observable de l'apprenant [BWA 94; THO 11]. Les approches cognitivistes et constructivistes mettent l'accent sur les activités cérébrales et postulent que les connaissances sont construites par l'apprenant via l'interaction avec l'environnement et la manipulation d'objets [PIA 1969]. Le socio-constructivisme y ajoute la dimension sociale : la construction des connaissances est médiatisée par les relations sociales [VYG 97]. Enfin, l'approche connectiviste voit l'apprentissage comme le développement d'un réseau reliant anciens et nouveaux savoirs [SIE 05].

Bien que différentes, ces approches ont pour trait commun l'attitude active de l'apprenant, une condition relevée par les récents travaux en neurosciences pour mieux comprendre les mécanismes cognitifs de l'apprentissage. Le progrès des techniques de neuro-imagerie (médicale) a fait avancer la compréhension du cerveau et les relations avec la cognition, donnant lieu à des projets scientifiques ambitieux comme le Human Brain Project dont l'objectif est de simuler le fonctionnement du cerveau ${ }^{2}$. Des facteurs clés ont ainsi pu être identifiés, servant de guide pour la mise en place d'activités d'apprentissage.

L'apprentissage se traduit chez l'individu par la création et la modification de connexions neuronales, une plasticité cérébrale qui doit être soutenue dès l'enfance par un environnement stimulant. Dans ses cours à la Chaire de psychologie cognitive expérimentale au Collège de France (année 2014-2015), Stanislas Dehaene expose les fondements cognitifs de l'apprentissage [DEH 14] ${ }^{3}$.

\footnotetext{
${ }^{1}$ Jiddu Krishnamurti, Apprendre est l'essence de la vie, Le Livre de Poche, Paris, 2010, 352p.

${ }^{2}$ Débuté en 2013 et s'étendant sur dizaine d'années, le Human Brain Project est soutenu par l'Union européenne et vise l'avancement de la recherche en neurosciences, informatique et médecine du cerveau par l'utilisation des TIC. (https://www.humanbrainproject.eu/en/, consulté le 16 décembre 2017)

${ }^{3}$ Depuis le 10 janvier 2018, le Conseil scientifique de l'Education nationale (France) présidé par Stanislas Dehaene assiste l'Education Nationale dans l'élaboration de méthodes pédagogiques en s'appuyant sur les résultats de la recherche scientifique, notamment en sciences cognitives. Source : (c) 2018 ISTE OpenScience - Published by ISTE Ltd. London, UK - openscience.fr Page | 2
} 
Il énonce quatre «piliers de l'apprentissage »: l'attention, l'engagement actif, le retour rapide d'informations et la consolidation quotidienne. Les scénarios d'apprentissage doivent susciter la curiosité, avec des matériaux attirants, pour captiver l'attention de l'apprenant. Cependant, l'intérêt et l'engagement cognitif pour ces matériaux ne doivent pas éclipser l'objet de l'apprentissage. L'engagement actif et le retour rapide d'informations sont étroitement liés à la théorie du «cerveau bayésien » décrivant le processus d'ajustement des représentations internes : (1) émettre une prédiction en se basant sur sa représentation interne actuelle ; (2) comparer la prédiction avec le monde extérieur ; (3) s'appuyer sur les erreurs pour ajuster sa représentation interne. L'apprenant doit être un participant actif dans les situations d'apprentissage et alterner avec des périodes de tests pour ajuster ses représentations internes. La consolidation de l'apprentissage passe par le sommeil, durant lequel les connaissances acquises sont transposées en automatismes inconscients, ainsi que par la répétition quotidienne des apprentissages. Finalement, les facteurs évoqués suggèrent la mise en place d'un apprentissage actif avec des situations attrayantes pour l'apprenant au cours desquelles il peut découvrir, expérimenter, se tester, dans un cadre délimité et avec un accompagnement adéquat.

L'identification des mécanismes de l'apprentissage et de ses ressorts cognitifs amène des modifications dans les méthodes d'apprentissage. Ces dernières évoluent également avec les technologies qui influencent le rapport aux savoirs et aux connaissances ainsi que nos outils de transmission.

\section{2. Évolution de l'apprentissage avec les technologies}

Michel Serres parle d'exo-darwinisme pour désigner le processus d'externalisation des fonctions corporelles induit par l'évolution technologique ${ }^{4}$. Il distingue quatre stades dans l'évolution du support d'information : l'oral, l'écriture manuscrite, l'imprimerie et l'ordinateur. À chaque changement, Serres constate une augmentation de la quantité d'informations et une plus grande facilité pour réaliser les quatre opérations de base sur celles-ci : stockage, traitement, émission et réception. Chaque stade se traduit par des transformations culturelles, politiques, religieuses et cognitives renouvelant les modes d'apprentissage et d'accès aux savoirs (voir figure 1).

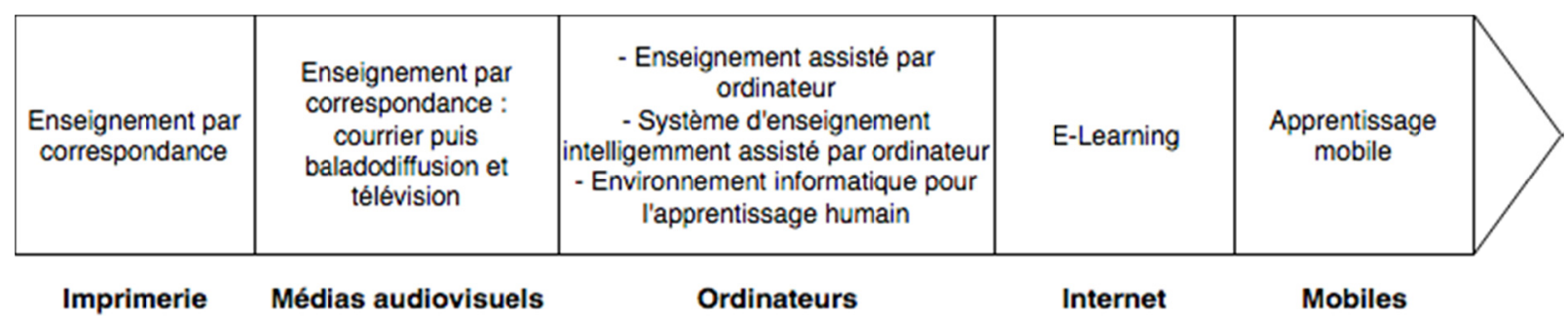

Figure 1. Évolution des modes d'apprentissage en fonction des supports d'information

Au stade oral, le corps humain constituait le support d'information puis, avec l'écriture manuscrite, le support est devenu pour la première fois externe à l'Homme. Avec l'imprimerie et l'essor des services postaux, l'enseignement par correspondance s'est développé, en premier lieu aux États unis au milieu et fin du $19^{\mathrm{e}}$ siècle [CAS 08]. À partir des années 1960, la téléphonie, la radio et la télévision ont complété les services postaux pour la transmission des cours [CAS 08]. Au quatrième stade, Serres voit dans l'ordinateur l'externalisation de la mémoire avec des capacités de stockage qui dépassent celles des stades antérieurs. La vaste quantité d'informations accessible depuis de multiples supports démultiplie les possibilités pour apprendre, un même concept étant abordable de plusieurs manières différentes pour s'adapter aux préférences de chacun (e.g. préférer la vidéo au texte, faire un jeu plutôt

\footnotetext{
https://www.lemonde.fr/education/article/2018/01/09/un-conseil-scientifique-pluridisciplinaire-pour-l-ecole 5239234 1473685.html, consultée le 26 janvier 2018.

${ }^{4}$ Conférence « Les nouvelles technologies : révolution culturelle et cognitive » par Michel Serres, à Lille en 2007.

(https://www.youtube.com/watch?v=ZCBB0QEmT5g, consulté le 12 décembre 2017).
} 
qu'étudier un schéma). L'informatique et les TIC élargissent les espaces pour apprendre et réduisent les contraintes temporelles, donnant ainsi naissance à l'apprentissage électronique (e-learning), suivi de l'apprentissage mobile (m-learning) avec l'amélioration des performances des appareils mobiles (e.g. mémoire, puissance de calcul, bande passante, couverture réseau).

Le développement de l'IdO s'inscrit dans la continuité de ces évolutions en externalisant non seulement la mémoire, mais aussi les capacités d'agir et d'interagir avec l'environnement. En s'appuyant sur l'apprentissage machine, les objets connectés remplacent les personnes pour certaines tâches quotidiennes grâce à des mécanismes d'automatisation et d'assistance. Ces changements requièrent de savoir se former pour suivre l'évolution des emplois et le renouvellement des besoins en compétences. En ce sens, dans une recommandation d'octobre $2006^{5}$, l'Union européenne met en avant huit compétences clés à maîtriser pour la formation tout au long de la vie : la langue maternelle ; les langues étrangères; les mathématiques, les sciences et les technologies pour la compréhension du monde ; l'utilisation des TIC pour la vie professionnelle et personnelle ; savoir se former ; participation à la vie sociale et démocratique ; savoir entreprendre ; l'ouverture culturelle. De par la pervasivité des objets connectés et leurs capteurs pour sonder le monde, l'IdO représente une opportunité pour l'apprentissage des sciences et la compréhension du monde.

\subsection{Dimensions caractéristiques de l'Internet des Objets}

Suivant le concept d'informatique ubiquitaire développé par Mark Weiser en 1988 pour désigner la fusion de l'informatique avec l'environnement [KRU 10], les TIC intègrent un nombre croissant d'objets qui deviennent des objets connectés. Ce foisonnement d'objets connectés est rendu possible par l'accroissement des puissances de calculs, le perfectionnement des batteries, la variété des technologies de communication, le cloud computing (l'informatique en nuage), la miniaturisation des composants, les Big Data et l'apprentissage machine [ROX 17]. Nous décrivons le fonctionnement et les caractéristiques de l'IdO à travers trois dimensions : données, interfaces et pervasivité (voir figure 2). Ce choix s'appuie sur un état de l'art mené sur les projets et applications concrètes de l'IdO ainsi que sur les travaux théoriques s'y rapportant.

L'IdO se forme à partir de l'interconnexion des objets, physiques (e.g. bracelet connecté) ou non (e.g. application de suivi d'activité physique), via le réseau Internet; ces objets sont identifiables de manière à pouvoir les contrôler ou les interroger (pour obtenir des données). L'exploitation des données issues des capteurs des objets connectés, leurs actuateurs pour agir sur l'environnement et leurs capacités de communication (avec l'utilisateur humain, d'autres objets ou des plateformes de services) constituent la base des applications de l'IdO.

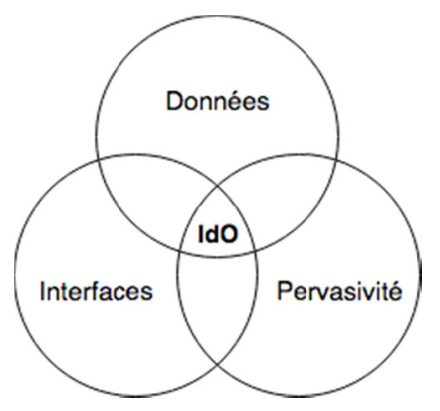

Figure 2. Les trois dimensions caractéristiques de l'IdO : données, interfaces et pervasivité.

Les données sont la première dimension caractéristique de l'IdO. Générées par des capteurs, cellesci correspondent à des grandeurs physiques telles que l'humidité d'une pièce, la pression, la température, la présence d'individus ou encore le taux de pollution dans l'air. Couplés avec

\footnotetext{
${ }^{5}$ Recommandation 2006/962/CE «L'éducation et la formation tout au long de la vie — Compétences clés ». Source : http://eur-lex.europa.eu/legalcontent/FR/TXT/?uri=LEGISSUM:c11090, consultée le 16 décembre 2017. 
l'apprentissage machine, les objets connectés acquièrent des capacités d'autonomie relative: les données qu'ils génèrent alimentent les algorithmes d'apprentissage qui produisent ensuite des modèles pour interpréter ces données en vue d'évaluer les situations, de prédire et d'agir (voir figure 3).

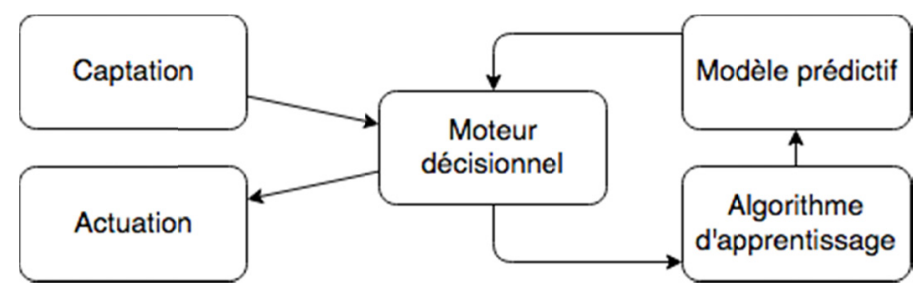

Figure 3. Exploitation des données des objets connectés avec l'apprentissage machine (adapté de [YAS 17])

Les interfaces et modes d'interaction des objets connectés constituent la deuxième dimension de l'IdO. L' ' enfouissement » des technologies dans les objets de l'environnement amène les concepteurs à adapter les interfaces homme-machine en fonction de la forme, de l'utilité ou encore de l'utilisabilité de l'objet. De cela, résultent des modes d'interaction plus naturels pour l'humain, interactions qui rapprochent les manières de contrôler et d'entrer les informations de leurs représentations (e.g. des pièces en bois connectées permettant de créer des formes dans une application pour tablette).

La pervasivité, dernière dimension du modèle, est d'ordre plus général et caractérise le milieu créé par l'IdO. Le mouvement d'enchevêtrement du numérique avec le physique tend à rendre les systèmes informatiques et les TIC ubiquitaires. Les objets connectés s'insinuent dans les lieux, les objets et les usages, créant ainsi des environnements imprégnés par le numérique.

Outre l'apprentissage machine nécessaire à l'interprétation des données, les spécificités des objets connectés renouvellent les modes de transmission de l'information et permettent de soutenir de nouvelles façons d'apprendre.

\section{Applications de l'IdO pour soutenir l'apprentissage humain}

Pour chacune des trois dimensions d'analyse évoquées précédemment (e.g. les données, les interfaces et la pervasivité), nous avons identifié plusieurs axes d'apprentissage, en regroupant les applications possibles de l'IdO pour soutenir l'apprentissage. Ces résultats sont synthétisés dans le tableau 1. 


\begin{tabular}{|l|l|l|l|}
\hline Dimension & \multicolumn{1}{|c|}{ Axe } & \multicolumn{1}{|c|}{ Apports potentiels } & \multicolumn{1}{c|}{ Exemples } \\
\hline \multirow{2}{*}{ Données } & $\begin{array}{l}\text { Comprendre le } \\
\text { monde }\end{array}$ & Contextualiser l'apprentissage des sciences. & $\begin{array}{l}\text { [ALI 17; JOY 14; MAE 15; PUT } \\
\text { 15] }\end{array}$ \\
\cline { 2 - 5 } & Adaptation & $\begin{array}{l}\text { Étendre le suivi et l'analyse de l'apprentissage au- } \\
\text { delà des plateformes de formation. }\end{array}$ & [CHE 12; NJE 17; TAA 14] \\
\hline \multirow{5}{*}{ Interfaces } & $\begin{array}{l}\text { Manipulation } \\
\text { d'objets }\end{array}$ & $\begin{array}{l}\text { Engager le corps, manipuler des concepts abstraits } \\
\text { et collaborer. }\end{array}$ & [FLE 17; GEO 17; KUB 16] \\
\cline { 2 - 5 } & $\begin{array}{l}\text { Environnement } \\
\text { augmenté }\end{array}$ & $\begin{array}{l}\text { Intégrer des informations dans les lieux et objets } \\
\text { de l'apprentissage et les rendre interactifs. }\end{array}$ & $\begin{array}{l}\text { [BAB 17; DIS 13; GON 08; KAL } \\
\text { 17; NAC 15; TAB 15] }\end{array}$ \\
\hline \multirow{2}{*}{ Pervasivité } & Mobilité & Dépasser les contraintes de temps et d'espace. & $\begin{array}{l}\text { [GIK 13; HUA 17; MAC 15; } \\
\text { SHA 09; WUW 12] }\end{array}$ \\
\cline { 2 - 5 } & Continuité & $\begin{array}{l}\text { Persistance de l'expérience d'apprentissage sur } \\
\text { plusieurs supports. }\end{array}$ & [MOU 17; NOR 13] \\
\cline { 2 - 5 } & Contexte & $\begin{array}{l}\text { Prendre en compte le contexte dans la } \\
\text { transmission des savoirs. }\end{array}$ & [HWA 09; KUH 16; SOU 15] \\
\hline
\end{tabular}

Tableau 1. Applications et activités possibles basées sur l'IdO pour soutenir l'apprentissage humain

Les catégories qui en découlent ne sont pas mutuellement exclusives et certaines applications s'inscrivent dans plusieurs axes.

\subsection{Données : informations sur les objets et les actions}

Les données issues des objets connectés servent l'apprentissage de deux façons : pour mieux comprendre le monde et pour adapter l'apprentissage au profil de l'apprenant et au contexte.

\subsubsection{Comprendre le monde}

Les données apportent des informations sur le monde : des grandeurs physiques caractérisant un écosystème ou le milieu d'un organisme vivant. Acquérir ces données est plus accessible aujourd'hui grâce à la baisse des coûts des composants et leur miniaturisation. Munis de capteurs, les objets connectés (e.g. téléphone mobile, tablette, montre connectée) et les kits de composants (e.g. Raspberry Pi ou Arduino) ${ }^{6}$ deviennent des outils de mesure. Couplés avec les technologies de communication dont ils disposent, ils permettent de visualiser les données à distance : un moyen de « voir » certaines réalités sans être présent physiquement.

Ces données favorisent un mode d'apprentissage situé [LAV 91], c'est-à-dire contextualisé par la mise en place d'une situation authentique et signifiante pour l'apprenant. Ce mode d'apprentissage rejoint les courants constructivistes et met l'accent sur l'environnement physique et social dans lequel la connaissance est construite. Les données portant sur l'environnement peuvent être employées pour l'apprentissage des sciences comme la physique ou les sciences de la vie et de la terre : les apprenants recueillent des données sur un environnement puis les analysent. Toutefois, les élèves peuvent rencontrer des difficultés pour interpréter les données [PUT 15] nécessitant un guidage.

Le projet Internet of School Things propose aux élèves des kits pour récolter des données [JOY 14] : par exemple, une station météo connectée pour étudier les saisons. Les auteurs appuient leur travail sur l'importance du contexte pour susciter l'intérêt des élèves pour la science. Il en va de même pour le

\footnotetext{
${ }^{6}$ L'article «Le Top 10 des kits de prototypage harware (3)» (2016) du magazine Makery propose une liste de plusieurs composants. (http://www.makery.info/2016/01/26/le-top-10-des-kits-de-prototypage-hardware-3/, consulté le 22 décembre 2017). 
prototype OBSY proposé par [PUT 15] et testé à travers des études sur le développement des plantes. [MAE 15] font état d'une expérimentation où les étudiants ont dû créer des prototypes pour collecter des données afin d'assurer la maintenance d'une serre. Ils montrent que le caractère authentique a amené les étudiants à collaborer et à échanger pour trouver des idées. Enfin [ALI 17] décrivent l'architecture de la plateforme IoTFLiP ${ }^{7}$ pour mettre à disposition d'étudiants en médecine des données de patients réels afin qu'ils puissent s'exercer. Ainsi, les étudiants ont accès à des cas authentiques et bénéficient de retours des médecins.

\subsubsection{Adaptation}

Les objets connectés renseignent sur les activités des individus en dehors de l'utilisation explicite d'outils numériques. Ces données étendent le champ d'action des techniques de traitement de données que sont l'analyse de l'apprentissage (learning analytics) et l'exploitation de données éducatives (educational data mining). L'analyse de l'apprentissage tend à personnaliser les conseils et les retours d'information à l'apprenant tandis que l'exploitation de données éducatives vise l'adaptation automatisée des systèmes d'apprentissage [SIE 12].

Quoi qu'il en soit, ces techniques s'inscrivent dans le cadre de l'apprentissage adaptatif dont l'objectif est d'individualiser l'expérience d'apprentissage en prenant en compte le profil de l'apprenant, son niveau de connaissances et ses activités antérieures. Avec l'IdO, le suivi de l'apprenant peut s'étendre au-delà du système d'apprentissage et intégrer ses déplacements et activités d'apprentissage informel: lire un livre, assister à une conférence, discuter avec un expert, etc. Toutefois, cette possibilité ne doit pas faire oublier les problématiques essentielles relatives à la vie privée et la sécurité des données personnelles ${ }^{8}$.

Dans [CHE 12], les auteurs décrivent un environnement pour l'apprentissage tout au long de la vie, utilisant des outils d'analyse de l'apprentissage. Le dispositif fournit, entre autres, des recommandations aux étudiants et enseignants, en exploitant les données d'objets connectés et d'une plateforme de formation. Les résultats des questionnaires montrent que les recommandations ont été reçues positivement par les étudiants et enseignants. Avec le même objectif, [NJE 17] proposent de coupler un cours en ligne à un réseau de capteurs pour identifier les scénarios d'apprentissage des apprenants. Plus techniquement, [TAA 14] s'appuient sur les objets connectés et l'ensemble de spécifications xAPI (Experience API, un standard pour l'enregistrement d'activités internes ou externes à un système d'apprentissage).

Nous avons vu que les objets connectés permettent d'appréhender le monde de par les données captées et le suivi des activités formelles et informelles des apprenants pour adapter l'apprentissage. Le lien avec l'environnement apporté par l'IdO se poursuit aussi au niveau des interfaces des objets connectés.

\subsection{Interfaces : interactivité des objets et lieux}

Les modes d'interaction homme-machine qui accompagnent les objets connectés apportent de nouveaux outils pour apprendre: par la manipulation d'objets concrets et en améliorant les environnements d'apprentissage.

\subsubsection{Manipulation d'objets}

Les interfaces des objets connectés tendent à se rapprocher des modes d'interaction naturels de l'humain. Aux verbes «taper», «pointer» et «cliquer» succèdent «toucher», «parler» et

\footnotetext{
${ }^{7}$ IoT- based flipped learning platform for medical education.

${ }^{8}$ A ce titre, le Parlement européen adopte le 14 avril 2016 le « règlement général sur la protection des données » pour protéger les données personnelles des individus au sein de l’Union européenne. Source :

https://fr.wikipedia.org/wiki/R\%C3\%A8glement_g\%C3\%A9n\%C3\%A9ral_sur_la_protection_des_donn\%C3\%A9es, consultée le 27 janvier 2018. 
«bouger ». Parmi ces interfaces utilisateur ubiquitaires (ubiquitous user interfaces), on distingue les interfaces tangibles (ou TUI pour «tangible user interfaces») et surfaciques (ou SUI pour «surface user interfaces ») [KRU 10]. Avec les TUI, les informations numériques sont palpables et prennent la forme d'objets physiques de telle sorte que manipuler un objet affecte une information. L'utilisateur pourra, par exemple, manipuler des pièces en bois représentant des lettres et, suivant leur positionnement, afficher un mot sur un écran. Quant aux interfaces surfaciques, ce sont des écrans ou des projections (horizontales ou verticales) comme les interfaces des téléphones mobiles, des tablettes, des tableaux blancs ou des tables interactives.

Dans le cadre de l'apprentissage, les interfaces tangibles et surfaciques diversifient les modes d'apprentissage en apportant des objets qui mobilisent le toucher. Elles permettent la mise en place d'activités pratiques où les interfaces tangibles donnent une forme concrète à un concept abstrait [REI 16]. Elles font écho à l'approche constructiviste de Piaget qui postule que le développement de la pensée passe par la manipulation d'objets, particulièrement chez l'enfant [KUB 16]. Comme le rappel [KUB 16], l'engagement corporel est également une composante recherchée par la pédagogie Montessori. Les interfaces surfaciques comme les tables tactiles ou interactives favorisent la collaboration : étant au même endroit, les apprenants peuvent se parler directement et voir les actions des autres (voir figure 4). Cependant, ces apports sont à nuancer, car des études sur l'apprentissage des mathématiques avec des objets palpables montrent qu'il ne semble pas y avoir de bénéfices significatifs [SCH 11]. D'autre part, les concepteurs doivent considérer la possibilité que l'apprenant applique les caractéristiques de l'objet au concept qu'il représente. Dans [SCH 11], les auteurs ajoutent que les connaissances ainsi acquises ne seraient accessibles à l'apprenant que par l'action, c'est-à-dire uniquement lors de la pratique.

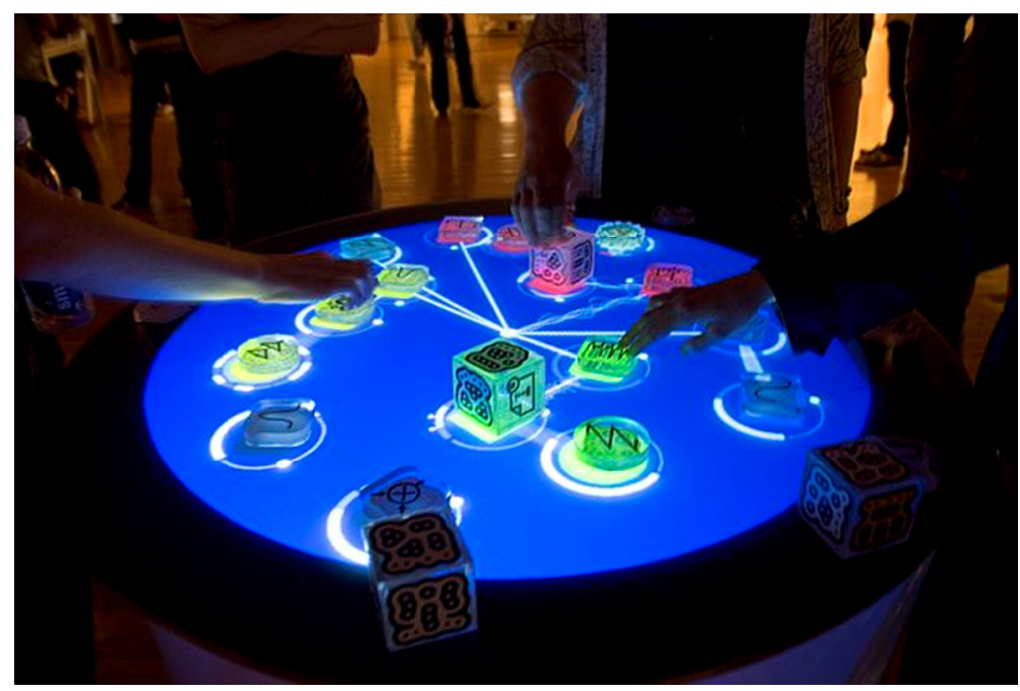

Figure 4. Collaboration autour d'une table interactive ${ }^{9}$

Les auteurs de [KUB 16] ont étudié le développement des «capacités de raisonnement spatial» chez des jeunes enfants avec des tables interactives munies d'objets tangibles. Ils mettent en évidence une amélioration des performances des élèves et leur collaboration pendant activités. Ces résultats se retrouvent aussi dans les expérimentations menées par [FLE 17; GEO 17] sur la compréhension des activités cérébrales et la programmation avec des interfaces tangibles. Outre les TUI, les applications Tynker $^{10}$ et CodeSpark ${ }^{11}$ s'appuient sur l'interface surfacique des tablettes pour l'apprentissage de la programmation en manipulant des objets correspondant à des instructions spécifiques. Ces applications s'inspirent de Scratch, un langage de programmation visuelle basé sur le langage Smalltalk et

\footnotetext{
${ }^{9}$ Image de Daniel Williams, https://commons.wikimedia.org/wiki/File:Reactable_Multitouch.jpg, consulté le 22 décembre 2017.

${ }^{10}$ https://www.tynker.com/, consulté le 29 novembre 2017.

${ }^{11} \mathrm{http}: / /$ codespark.org/, consulté le 29 novembre 2017.

(C) 2018 ISTE OpenScience - Published by ISTE Ltd. London, UK - openscience.fr 
l'environnement de programmation Squeak ${ }^{12}$. Distribué depuis 2003 par le groupe de recherche LifeLong Kindergarten du MIT MediaLab avec le slogan « Imagine, Programme, Partage », Scratch permet une approche ludique de l'algorithmique et vise à initier les élèves aux fondements des mathématiques et de la programmation ${ }^{13}$. Les élèves sont invités à imaginer et créer des histoires interactives et des jeux en manipulant des blocs de code. Depuis 2014, Tynker reprend ces principes dans une application pour tablettes où les utilisateurs peuvent glisser-déposer les blocs de code. La figure 5 présente un exemple de programme réalisé avec Tynker ${ }^{14}$.
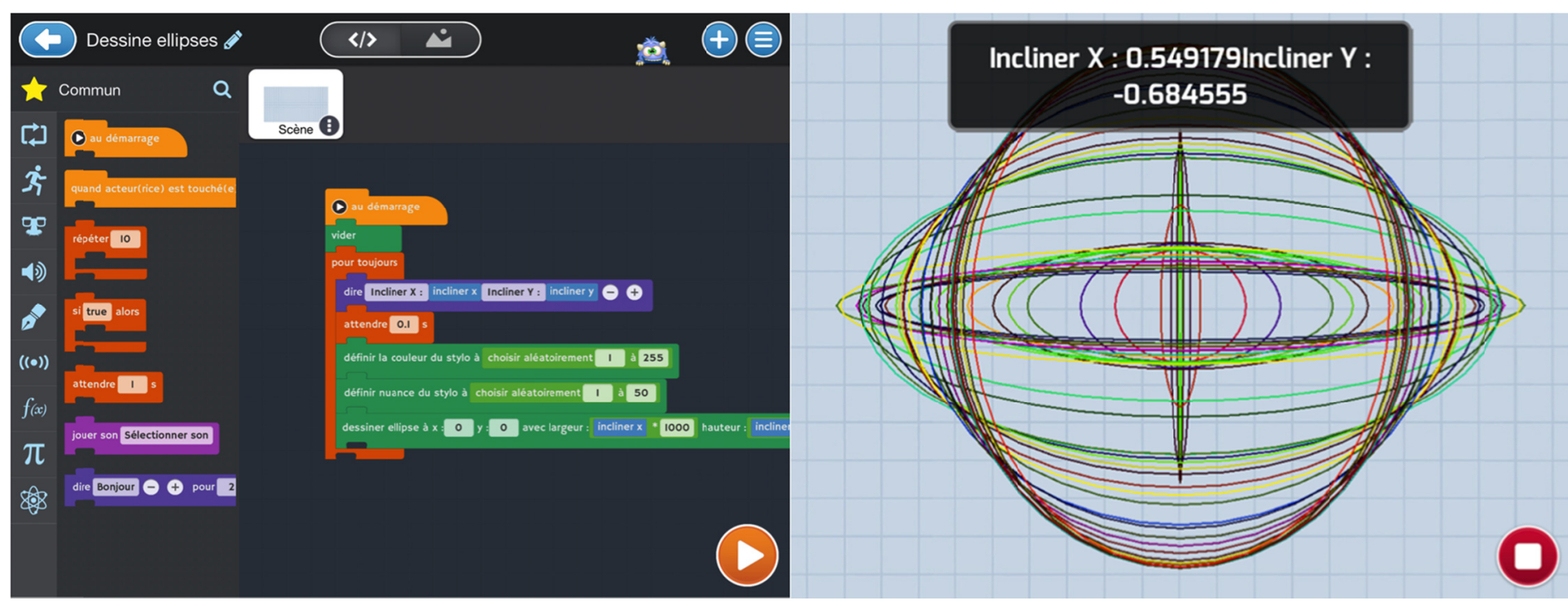

Figure 5. Exemple de programmation visuelle avec Tynker : les blocs d'instructions (à gauche) permettent de dessiner (à droite) des ellipses dont les largeurs et hauteurs varient en fonction de l'inclinaison de la tablette.

Certains jouets éducatifs connectés combinent les interfaces tangibles et surfaciques pour proposer plus de fonctions, tels que les robots Cozmo ${ }^{15}$ et Robot Dash $^{16}$ qui sont programmables depuis une tablette à la manière de Tynker et CodeSpark.

\subsubsection{Environnement augmenté}

L'IdO apporte une couche interactive à l'environnement avec le déploiement de la réalité augmentée et les interfaces ambiantes utilisant par exemple les étiquettes RFID (Radio Frequency Identification) et NFC (Near Field Communication) et les capteurs. À l'inverse des interfaces tangibles et surfaciques, les interfaces ambiantes ne requièrent pas l'engagement de l'utilisateur. Elles affichent des informations à l'utilisateur dans la périphérie du spectre d'attention de l'utilisateur et celui-ci peut se concentrer dessus lorsqu'il le souhaite [KRU 10]. Les LEDs des chargeurs d'ordinateur indiquant la présence ou non de courant sont un exemple d'interface ambiante.

Les étiquettes RFID et NFC interrogeables par les téléphones mobiles peuvent contribuer à rendre les lieux d'apprentissage plus pratiques en intégrant des informations (e.g. horaires de secrétariat, ressources éducatives, coordonnées d'enseignant, notice d'un matériel) dans des objets physiques. Ces informations combinées à celles fournies par les capteurs sur les lieux et matériels d'apprentissage, peuvent rendre accessibles à distance ces lieux et les matériels d'apprentissage. De plus, les espaces peuvent être adaptés aux usages et habitudes des étudiants en exploitant les données recueillies par des capteurs. La réalité augmentée ajoute une aide contextuelle en lien avec un lieu ou une activité : à

\footnotetext{
${ }^{12}$ Conçu initialement au Xerox PARC, puis Apple et Disney Interactive, Squeak est un environnement de programmation dynamique dédié à l'origine aux applications éducatives, notamment comme espace de créativité. (https://wikipedia.org/wiki/Squeak).

${ }^{13} \mathrm{https}$ ://en.wikipedia.org/wiki/Scratch (programming language), consulté le 27 janvier 2018.

14 https://en.wikipedia.org/wiki/Tynker, consulté le 27 janvier 2018.

$15 \mathrm{https} / / /$ www.anki.com/fr-fr/cozmo, consulté le 27 janvier 2018.

16 https://www.makewonder.com/dash, consulté le 27 janvier 2018.
} 
travers l'écran d'appareils mobiles, les utilisateurs voient une réalité à laquelle sont superposées des informations calculées en temps réel par un programme.

L'usage d'étiquettes NFC pour distribuer des informations aux étudiants est proposé par [GON 08; TAB 15]. Les auteurs [TAB 15] rapportent plusieurs scénarios d'utilisation de la technologie NFC, notamment pour l'apprentissage formel : échange de documents entre enseignants et étudiants, ajout d'informations aux documents imprimés. Dans [KAL 17], les auteurs proposent des prototypes pour rendre accessibles à distance les équipements d'un laboratoire. Les travaux de [DIS 13; NAC 15] sur les bénéfices de la réalité augmentée pour l'apprentissage montrent une augmentation de la motivation auprès des étudiants. [BAB 17] rapporte des exemples d'applications possibles de la réalité augmentée pour l'éducation : un tableau virtuel sur lequel les élèves peuvent écrire avec un pointeur laser, un livre papier augmenté par des contenus vidéo ou des animations et un jeu pour appareil mobile.

Les données et les interfaces des objets connectés traduisent l'imbrication du numérique avec l'environnement opéré par l'IdO et constituent des opportunités pour comprendre le monde via des activités authentiques ainsi que des lieux et objets interactifs. Englobant les données et les interfaces, la pervasivité de l'IdO réduit les contraintes de temps, d'espace et de support lié à l'apprentissage.

\subsection{Pervasivité : contextualisation des activités}

La pervasivité des objets connectés et la couverture croissante du réseau Internet permettent le développement de systèmes d'apprentissage en tenant compte de trois aspects : la mobilité, la continuité et la sensibilité au contexte.

\subsubsection{Mobilité}

La lecture de contenus multimédia et leur téléchargement depuis des objets connectés sont rendus possibles grâce à l'amélioration des performances (e.g. mémoire, puissance de calcul), de la couverture du réseau cellulaire et des débits de transfert de données. Ce faisant, les téléphones mobiles et tablettes concurrencent les ordinateurs fixes et portables, réduisant les contraintes de temps et d'espace qui prévalaient.

Pour l'apprentissage, les objets connectés deviennent des outils multifonctions pour soutenir des activités diverses: réalisation d'albums photo, montage vidéo, enregistrement audio, recherche d'informations, etc. Le fort taux d'équipement en téléphone mobile chez les jeunes générations permet la pratique du BYOD (Bring Your Own Device - "Apportez votre propre appareil ») : les élèves et étudiants sont invités à utiliser leurs propres appareils, évitant ainsi les coûts liés à l'achat de matériel pour les écoles et les universités [VAN 12]. Toutefois, ce type de pratique révèle les inégalités d'équipements qui peuvent exister entre les apprenants. Le e-learning évolue également vers l'apprentissage mobile: les activités et applications sont transposées aux téléphones mobiles et tablettes. Ces objets connectés investissent aussi l'apprentissage informel à travers le microlearning qui permet d'introduire des exercices pour apprendre dans les moments d'attente de la vie quotidienne. Les formations sont alors divisées en unité réalisable en quelques minutes [JOM 16].

De nombreuses recherches ont été entreprises sur l'apprentissage mobile, la majorité portant sur les potentiels apports et présentant effectivement des résultats positifs [WUW 12]. L'approche qualitative élaborée par [GIK 13] met en avant quatre avantages pour les apprenants : a) l'accès rapide aux informations, b) la communication et la collaboration, c) la diversité des formes des activités et d) l'apprentissage situé. La co-construction des connaissances et l'apprentissage dans des situations authentiques sont également pointés par [SHA 09] en évoquant les possibilités d'exploration permises par les appareils mobiles. [MAC 15] ont étudié l'utilisation faite des objets connectés par un enseignant en jardin d'enfants et montrent qu'ils sont employés pour s'adapter aux besoins et intérêts des enfants (e.g. création d'un livre de photos, création d'un e-book mélangeant textes et images). Enfin, [HUA 17] rapportent une expérimentation basée sur un jeu sérieux pour l'apprentissage du vocabulaire 
anglais. À partir de questionnaires, les auteurs rapportent des résultats positifs sur la motivation et la satisfaction des étudiants, mais mettent en évidence le manque d'expérience des étudiants vis-à-vis de ce mode d'apprentissage.

En France, le ministre de l'Éducation nationale a confirmé ${ }^{17}$ l'interdiction à l'école et au collège des téléphones portables à la rentrée 2018 . Une telle mesure ${ }^{18}$ vise à favoriser la concentration des élèves et lutter contre la triche. Les avis sont partagés. Effectivement, sans une vraie stratégie pédagogique et une sérieuse formation des enseignants pour la maîtrise des outils numériques, l'intérêt pédagogique des téléphones portables est limité. Comme évoqué à travers les différents exemples, les téléphones portables, en tant qu'objets connectés, peuvent être exploités de manière constructive au collège (e.g. recherches documentaires et de définitions en français; photos d'expériences, chronomètre ou boussole, en physique ; partage de documents multimédias en anglais). Ainsi impliqué, l'élève devient l'acteur de la construction du savoir et l'enseignant peut assurer un enseignement personnalisé. Les choses ne sont ni simples ni faciles, mais à long terme il sera difficile d'interdire à la vie réelle de rentrer à l'école. Au lieu d'interdire, il serait préférable de responsabiliser et de stimuler une vraie réflexion sur l'évolution de l'éducation vers une sage utilisation du numérique. Si l'interdiction des téléphones portables peut stimuler l'attention des élèves en classe, il n'est pas du tout certain que leur motivation augmentera. Cette motivation est plutôt liée à la capacité des professeurs à transmettre l'amour de la connaissance. À son tour, cette capacité est stimulée par plusieurs facteurs : passion, motivation et talent, mais aussi salaire et reconnaissance sociale. Stimuler ces facteurs est plus important que bloquer la technologie dans les écoles.

\subsubsection{Continuité}

L'étude «How People Use Their Devices - What Marketers Need to Know » menée par Google en 2016 montre que 57\% des utilisateurs effectuent des recherches à partir de plusieurs appareils différents [GOO 16]. Avec le développement de l'IdO, les individus tendent à jongler avec plusieurs objets connectés pour utiliser les services et outils numériques. Une évolution permise notamment par le cloud computing qui assure la synchronisation des données des utilisateurs d'un appareil à l'autre.

Tenant compte de cela, il est possible de démarrer un cours de programmation sur son téléphone mobile le matin en attendant le bus, poursuivre le midi sur son ordinateur portable au bureau et terminer le cours le soir sur sa tablette chez soi. L'expérience d'apprentissage est continue et traverse les supports sans perte d'informations.

Plusieurs applications intègrent cet aspect comme Tynker ou Duolingo ${ }^{19}$ pour l'apprentissage des langues. L'expérience d'apprentissage proposée est adaptée en fonction des spécificités de chaque appareil. [NOR 13] ont étudié les usages d'objets connectés par des étudiants et montrent que l'un des aspects les plus recherchés est l'accès ubiquitaire aux ressources. La continuité de l'expérience d'apprentissage se situe aussi entre les apprentissages formel et informel. [MOU 17] décrivent un système d'analyse et de visualisation de traces d'apprentissage formel et informel. Dédié à l'apprentissage des langues, VASCROLL (Visualization and Analysis System for Connecting Relationships of Learning Logs) permet d'enregistrer, d'organiser, de rappeler et d'évaluer les mots et expressions apprises. Les apprenants peuvent suivre et évaluer leur apprentissage.

\footnotetext{
${ }^{17}$ http://www.lemonde.fr/education/article/2017/12/11/1-interdiction-du-telephone-portable-a-l-ecole-une-mesure-difficilementapplicable $5228060 \quad 1473685 . h$ tml

${ }^{\frac{1}{18}}$ A New York, l'interdiction des téléphones portables dans les écoles de la ville a été imposée en 2006 et levée en 2015. Les adeptes de « digital detox » en se basant sur l'augmentation du cyberharcèlement sollicitent à interdire les portables à nouveau. En Belgique, depuis la rentrée 2017, une vingtaine d'établissements testent la pratique du BYOD. En Italie, le téléphone portable est strictement interdit en classe depuis 2007, mais en septembre 2017 la ministre de l'éducation s'est prononcée en faveur d'un usage pédagogique en parlant même d'une " opportunité pour l'apprentissage » (http://www.lemonde.fr/societe/article/2017/12/14/1-interdiction-des-telephones-portables-a-1-ecole-fait-aussi-debat-a-letranger_5229851_3224.html\#lC4zmbZelGebmBkd.99).

${ }^{19} \mathrm{http} / / /$ fr.duolingo.com, consulté le 26 janvier 2018.
} 


\subsubsection{Contexte}

Par le biais de leurs capteurs, les objets connectés permettent de développer des systèmes sensibles au contexte, par exemple : distribuer des publicités ciblées, diffuser une vidéo relative à un lieu ou informer les citoyens en situation post-attentat. Dans sa thèse, [SOU 15] étaye les dimensions du contexte : temps, espace, dispositif, environnement, utilisateur et activité. Toutes ces variables sont prises en compte par les systèmes informatiques.

Les systèmes dédiés à l'apprentissage adaptent les contenus ou les conseils en fonction de la situation réelle de l'apprenant. En ce sens, les systèmes d'apprentissage sensible au contexte favorisent l'apprentissage situé : le rapprochement entre la situation-problème et l'activité d'apprentissage (e.g. apprendre à réparer son vélo en étant à côté). Plus globalement, la mobilité, la continuité et la prise en compte du contexte sont les composantes de l'apprentissage ubiquitaire : un mode d'apprentissage caractérisé par la capacité à apprendre la bonne chose, au bon endroit, au bon moment et de la bonne façon [YAH 10].

Parmi les travaux intégrant le contexte, [KUH 16] rapportent une expérimentation avec des Google Glass pour illustrer sous la forme d'un graphique la relation entre la masse d'un verre d'eau et la fréquence de vibration selon le niveau de remplissage. Les auteurs montrent que cela stimule la curiosité des étudiants sans pour autant augmenter la charge cognitive. Dans [HWA 09], les auteurs proposent un système d'apprentissage ubiquitaire pour aider les jeunes chercheurs pour la réalisation d'expériences. Les interviews semi-structurés menés par les auteurs montrent que le système est un gain de temps pour les chercheurs et permet d'apprendre au plus près du problème. Dans un autre domaine, [SOU 15] propose l'architecture d'un système d'apprentissage sensible au contexte dédié à la formation des employés d'une entreprise.

L'IdO fournit un cadre d'apprentissage orienté vers l'exploration, l'expérimentation et l'adaptation - au contexte et à l'apprenant. L'apprenant est amené à interpréter et à interagir dans des activités construites autour des situations authentiques, avec des matériaux tangibles. Ces conditions se prêtent notablement à l'apprentissage des sciences, des mathématiques, de la physique et aux sciences de la vie et de la terre en passant par l'ingénierie et les technologies. Cependant, dans un contexte de diversité abondante de ressources d'apprentissage, les compétences informationnelles des individus sont primordiales pour rechercher, identifier et utiliser ces ressources [KEN 17]. Plusieurs standards ont été élaborés pour documenter les ressources numériques d'apprentissage et soutenir le développement de système de recherche et d'identification (e.g. Learning Object Metadata, DublinCore Metadata Initiative). Toutefois ces approches documentaires nécessitent d'être revues pour prendre en compte la nouvelle diversité de ressources naissant avec l'IdO.

\section{Conclusion}

Dans cet article, nous avons examiné la relation entre l'IdO et l'apprentissage humain en nous appuyant sur les trois dimensions de l'IdO (données, interfaces et pervasivité) pour étayer les différentes applications et activités qui peuvent être mises en œuvre pour soutenir l'apprentissage. Les travaux en neurosciences cognitives ont permis d'identifier certains facteurs clés de l'apprentissage humain: l'attention, l'engagement actif, le retour rapide d'informations et la consolidation quotidienne. Ces ressorts cognitifs se couplent avec les technologies : après l'imprimerie, les médias audiovisuels, l'ordinateur et Internet, les objets connectés constituent les nouveaux outils de transmission des savoirs. Plus concrètement, l'IdO permet la mise en place de situations stimulantes pour les apprenants en favorisant l'exploration et l'expérimentation dans des situations authentiques. Les applications de l'IdO pour l'apprentissage s'inscrivent dans la réalité et le tangible à travers des capteurs mesurant l'environnement, des interfaces rendant l'information palpable et les lieux interactifs, ainsi que la réduction des contraintes de temps, d'espace et de support. De plus, 
l'expérience d'apprentissage traversant les supports, les apprentissages formels et informels tendent à se rejoindre et les plateformes de formation deviennent sensibles au contexte.

Toutefois, la relation entre IdO et apprentissage ne se limite pas aux humains. Sous l'apparence de chaussures, de lunettes, de stylos, de réveils, de montres, de bracelets, de tables et de drones, les machines implémentent des mécanismes d'apprentissage augmentant les capacités de ces objets avec des fonctions d'automatisation et d'assistance simulant les comportements humains. Les systèmes apprenants s'appuient sur des formalismes algorithmiques inspirés des neurosciences (e.g. réseau de neurones, système attentionnel, mémoires de travail et épisodique) pour améliorer leurs performances. En étudiant de grands volumes de données pour déceler des relations et établir un modèle, les systèmes apprenants peuvent émettre des prédictions et évaluer des situations à partir de nouvelles données. Dans un prochain article, nous montrerons que, pour "comprendre» le monde, les systèmes informatiques implémentent des mécanismes cherchant à reproduire les capacités d'apprentissage des humains, mais dans des proportions et avec des méthodes différentes.

\section{Références}

[ALI 17] Ali, M., BILAL, H.S.M., RAZZAQ, M.A., ET AL. IoTFLiP: IoT-based flipped learning platform for medical education. Digital Communications and Networks 3, 3, 188-194, 2017.

[BWA 94] B. WATson, J. Psychology as the Behaviorist Views It. Psychological Review 101(2), p.248-253, 1994.

[BAB 17] BABU, N.S.C. Keynote 1: Internet of Things(IoT) and augmented reality for e-learning. 2017 5th National Conference on E-Learning E-Learning Technologies (ELELTECH), 1-10, 2017.

[CAS 08] CASEY, D.M. A Journey to Legitimacy: The Historical Development of Distance Education through Technology. TechTrends 52, 2, 45, 2008.

[CHE 12] CHENG, H.C. AND LIAO, W.W. Establishing an lifelong learning environment using IOT and learning analytics. 2012 14th International Conference on Advanced Communication Technology (ICACT), 1178-1183, 2012.

[DEH 14] DeHAENE, S. Fondements cognitifs des apprentissages scolaires. Collège de France. http://www.college-defrance.fr/site/stanislas-dehaene/course-2014-2015.htm, 2014.

[DIS 13] Di SERIO, Á., IBÁÑEZ, M.B., AND KLOOS, C.D. Impact of an augmented reality system on students' motivation for a visual art course. Computers \& Education 68, 586-596, 2013.

[FlE 17] Fleck, S., Baraudon, C., Frey, J., Laine, T., AND Hachet, M. «Teegi, il est trop beau»: Exemple d'évaluation du potentiel pédagogique d'une interface tangible interactive pour enfants en contexte scolaire. 29ème conférence francophone sur l'Interaction homme-machine, 12-p., 2017.

[GeO 17] George, S., Marfisi-Schottman, I., And Leconte, M. TurtleTable: apprendre les bases de la programmation avec des interfaces tangibles. Journée d'étude "IHM pour l'éducation », Poitiers, 2017.

[GIK 13] GIKAS, J. AND GRANT, M.M. Mobile computing devices in higher education: Student perspectives on learning with cellphones, smartphones \& social media. The Internet and Higher Education 19, Supplement C, 18-26, 2013.

[GON 08] GONZÁLEZ, G.R., ORGANERO, M.M., AND KloOs, C.D. Early Infrastructure of an Internet of Things in Spaces for Learning. IEEE, 381-383, 2008.

[GOO 16] GoOgle. How People Use Their Devices - What Marketers Need to Know. https://storage.googleapis.com/think/docs/twg-how-people-use-their-devices-2016.pdf, septembre 2016.

[HUA 17] HuAng, Y.-L., Chang, D.-F., AND WU, B. Mobile Game-Based Learning with a Mobile App: Motivational Effects and Learning Performance. Journal of Advanced Computational Intelligence and Intelligent Informatics 21, 6 , 963-970, 2017.

[HWA 09] HWANG, G.-J., YANG, T.-C., TSAI, C.-C., AND YANG, S.J.H. A context-aware ubiquitous learning environment for conducting complex science experiments. Computers \& Education 53, 2, 402-413, 2009.

[JOM 16] JOMAH, O., MASOUd, A.K., KiShORE, X.P., AND AURELIA, S. Micro learning: A modernized education system. BRAIN. Broad Research in Artificial Intelligence and Neuroscience 7, 1, 103-110, 2016.

[JOY 14] Joyce, C., Pham, H., Stanton Fraser, D., Payne, S., Crellin, D., And McDougall, S. Building an internet of school things ecosystem: a national collaborative experience. ACM Press, 289-292, 2014. 
[KAL 17] KALASHNiKOV, A., ZHANG, H., JENNINGS, J., AND ABRAMrIUK, M.-M. Remote laboratory: using Internet-ofThings (IoT) for E-learning. The Vth International Conference "Advanced Information Systems and Technologies, 2017.

[KEN 17] KenNel, S. AND ChEVRY PeBAYle, E. Les pratiques et apprentissages informationnels nformimpensé des EIAH? Actes de la 8e édition de la conférence EIAH, 149-159, 2017.

[KRI 10] KrishnamuRTI, J. Apprendre est l'essence de la vie. Le Livre de Poche, Paris, 2010.

[KRU 10] KRUMM, J., ED. Ubiquitous computing fundamentals. Chapman \& Hall/CRC Press, Boca Ragon, 2010.

[KUB 16] Kubicki, S., Pasco, D., HoAreau, C., AND Arnaud, I. Utilisation d'une Table Interactive avec objets Tangibles pour apprendre à l'école: études empiriques en milieu écologique. Actes de la 28ième conférence francophone sur l'Interaction homme-machine, 155-166, 2016.

[KUH 16] Kuhn, J., LuKowicz, P., Hirth, M., POXrucker, A., WePPner, J., AND YounAS, J. gPhysics-Using Smart Glasses for Head-Centered, Context-Aware Learning in Physics Experiments. IEEE Transactions on Learning Technologies 9, 4, 304-317, 2016.

[LAV 91] LaVe, J. AND Wenger, E. Situated Learning: Legitimate Peripheral Participation. Cambridge University Press, 1991.

[MAC 15] MacCALlum, K. AND Bell, H. Smart devices for supporting inquiry and conversations in early childhood education. He Kuрu Word 4, 1, 31-39, 2015.

[MAEN 15] MäenpäÄ, H., TARKoma, S., VArjonen, S., And Vihavainen, A. Blending Problem- and Project-Based Learning in Internet of Things Education: Case Greenhouse Maintenance. Proceedings of the 46th ACM Technical Symposium on Computer Science Education, ACM, 398-403, 2015.

[MOR 92] MORIN, E. La méthode. Tome III. La connaissance de la connaissance. Anthropologie de la connaissance. Editions du Seuil, 1992.

[MOR 08] Morin, E. La méthode. Vol. I, Editions du Seuil, 2008.

[MOU 17] MOURI, K., OGATA, H., AND UOSAKI, N. Learning analytics in a seamless learning environment. ACM Press, 348-357, 2017.

[NAC 15] NACHAIRIT, A. AND SRISAWASDI, N. Using Mobile Augmented Reality for Chemistry Learning of Acidbase Titration: Correlation between Motivation and Perception. The $23^{\text {rd }}$ International Conference on Computers in Education, China, 2015.

[NJE 17] NJERU, A.M., OMAR, M.S., AND YI, S. IoTs for capturing and mastering massive data online learning courses. 2017 IEEE/ACIS 16th International Conference on Computer and Information Science (ICIS), 91-94, 2017.

[NOR 13] NortCliffe, A. AND Middleton, A. The Innovative Use of Personal Smart Devices by Students to Support their Learning. In: L.A. Wankel and P. Blessinger, eds., Cutting-edge Technologies in Higher Education. Emerald Group Publishing Limited, 175-208, 2013.

[PIA 69] Piaget, J. Psychologie et pédagogie. Paris, France, 1969.

[PUT 15] PUTJORn, P., ANG, C.S., AND FARZIN, D. Learning IoT Without the "I"- Educational Internet of Things in a Developing Context. Proceedings of the 2015 Workshop on Do-it-yourself Networking: An Interdisciplinary Approach, ACM, 11-13, 2015.

[REI 16] REIMANN, D. AND MADAY, C. Smart textile objects and conductible ink as a context for arts based teaching and learning of computational thinking at primary school. ACM Press, 31-35, 2016.

[ROX 17] RoXIn, I. AND BOUCHEREAU, A. Ecosystème de l'Internet des Objets. In: Internet des Objets. Paris, France, 2017.

[SAL 17] SALEH, I., 2017. « Les enjeux et les défis de l'Internet des Objets (IdO) », Revue «Internet des objets » 1. DOI:10.21494/ISTE.OP.2017.0133

[SCH 11] Schneider, B., Jermann, P., Zufferey, G., AND Dillenbourg, P. Benefits of a Tangible Interface for Collaborative Learning and Interaction. IEEE Transactions on Learning Technologies 4, 3, 222-232, 2011.

[SHA 09] Sharples, M., ARnedillo-SÁnChez, I., Milrad, M., AND VAvoula, G. Mobile Learning. In: TechnologyEnhanced Learning. Springer, Dordrecht, 233-249, 2009.

[SIE 05] SIEMENS, G. Connectivism: A Learning Theory for the Digital Age. International Journal of Instructional Technology \& Distance Learning 2, 1, 2005. 
[SIE 12] SIEMENS, G. AND D BAKER, R.S. Learning analytics and educational data mining: towards communication and collaboration. Proceedings of the 2nd international conference on learning analytics and knowledge, ACM, 252-254, 2012.

[SOU 15] Soualah-AliLA, F. CAMLearn: Une Architecture de Système de Recommandation Sémantique Sensible au Contexte. Application au Domaine du M-Learning. https://hal.archives-ouvertes.fr/tel-01238233/, 2015.

[TAA 14] TAamallah, A. AND KhemaJA, M. Designing and eXperiencing smart objects based learning scenarios: an approach combining IMS LD, XAPI and IoT. ACM Press, 373-379, 2014.

[TAB 15] TabuencA, B., Kalz, M., And SPeCht, M. Tap it again, Sam: Harmonizing Personal Environments towards Lifelong Learning. International Journal of Advanced Corporate Learning (iJAC) 8, 1, 16, 2015.

[THO 11] THORNDIKE, E.L. EdWARD L. Animal intelligence; experimental studies. New York, The Macmillan company, 1911.

[VAN 12] VANWELSENAERS, M. Students using their own technology device in the classroom: Can "BYOD" increase motivation and learning. Unpublished Masters of Arts, Northern Michigan University, Marquette, 2012.

[VYG 97] VyGOTSKI, L.S. Pensée et langage. Paris, France, 1997.

[WUW 12] WU, W.-H., JIM WU, Y.-C., CHEN, C.-Y., KAO, H.-Y., LIN, C.-H., AND HUANG, S.-H. Review of trends from mobile learning studies: A meta-analysis. Computers \& Education 59, 2, 817-827, 2012.

[YAH 10] YAHYA, S., ARNIZA AhMAD, E., AND ABD JALIL, K. The definition and characteristics of ubiquitous learning: A discussion. International Journal of Education and Development using Information and Communication Technology (IJEDICT) 6, 1, 117-127, 2010.

[YAS 17] YASHESH SHROFF. Machine Learning in IoT Applications - Global IoT Devfest With The Best 2017. https://www.youtube.com/watch?v= RYZHxKiCIE\&feature=youtu.be, 2017. 